\title{
Lung function effects of chronic exposure to air pollution
}

\section{Jordi Sunyer}

Lung function is an excellent operative marker of the effects of air pollution in the general population. It is objective and quantitative, an early predictor of cardiorespiratory morbidity and mortality, able to describe trajectories to the occurrence of chronic obstructive pulmonary disease (COPD) and coherent with experimental data on deposition and accumulation of pollutants in airways and lungs and the resulting systemic inflammation and oxidative stress. ${ }^{12}$

Acute effects of air pollution are well established, whereas long-term effects are less certain. There is strong evidence of long-term effects of current levels of air pollution on lung function growth in children, resulting in deficits of lung function at the end of adolescence. ${ }^{3}$ However, there are no data about the potential reversibility of the deleterious effect afterwards. In adults, there is only one large long-term follow-up study. ${ }^{4}$ The strongest evidence for adverse long-term effects of air pollution on lung function in adults comes from cross-sectional investigations. In Europe, these studies were limited to a few residential areas. ${ }^{56}$

Forbes et al have published in this issue (see page 657) the largest cross-sectional study in adults in Europe, comprising four English surveys conducted between the years 1995 and 2001 in 648-720 postcode areas, adding 41329 subjects older than age 16 and living in around 30000 households. ${ }^{7}$ Individual residential exposure to air pollutants was based on dispersion modelling including meteorological and emission data for each postcode. Air pollution levels are moderate (median particulate matter $<10 \mu \mathrm{m}\left(\mathrm{PM}_{10}\right)$ and $\mathrm{NO}_{2}$ around 20 and $30 \mu \mathrm{g} / \mathrm{m}^{3}$, respectively). An increase in $10 \mu \mathrm{g} / \mathrm{m}^{3}$ of $\mathrm{PM}_{10}$ and $\mathrm{NO}_{2}$ was associated with a decrease of about $3 \%$ and $0.7 \%$ in $\mathrm{FEV}_{1}$ (forced expiratory volume in $1 \mathrm{~s}$ ), respectively. This may imply an increase of around $50 \%$ of the population with an $\mathrm{FEV}_{1}$ of $<80 \%$.

The size of the effects in the English study is similar to that of the other two

Correspondence to: Dr Jordi Sunyer, Center of Research of Environmental Epidemiology (CREAL), IMIM, Barcelona, Catalonia, Spain; jsunyer@creal.cat
European multicentre studies. The Swiss Study on Air Pollution and Lung Disease in Adults (SAPALDIA) in 9651 subjects (18-60 years of age) across eight study communities found that an increase of $10 \mu \mathrm{g} / \mathrm{m}^{3}$ in annual mean concentration of $\mathrm{PM}_{10}$ was associated with a $3.4 \%$ decrease in forced vital capacity (FVC) and a $1.6 \%$ decrease in $\mathrm{FEV}_{1} .{ }^{5}$ The German study on the Influence of Air Pollution on Lung Function, Inflammation, and Aging (SALIA), including 2593 women with a mean age of 54.5 years across seven communities, showed significant negative associations of $\mathrm{NO}_{2}$ and $\mathrm{PM}_{10}$ with $\mathrm{FEV}_{1}$, FVC and the $\mathrm{FEV}_{1} / \mathrm{FVC}$ ratio $(4.7 \%, 3.4$ and 1.1 per $10 \mu \mathrm{g} / \mathrm{m}^{3} \mathrm{PM}_{10}$, respectively). ${ }^{6}$

A compatible size of the effects between the three studies was obtained using different strategies to estimate the individual measurement of the average chronic exposure to air pollution. The three studies assign an individual estimation to each participant based on their home address and using dispersion models. The spatial resolution varies from $8 \mathrm{~km}^{2}$ in SALIA, to $1 \mathrm{~km}^{2}$ in the English study and to $200 \mathrm{~m}^{2}$ in SAPALDIA. Both SALIA and SAPALDIA used data from monitoring stations, the former combined with distance to the nearest major road, while the English study used emission data from multiple sources. This makes the measurement of the English study more precise since the proportion of subjects living close to major roads is small. However, in contrast to SAPALDIA which estimates the individual assignment according to the residential history, the English study was limited to the residence at the time of the survey. It is very unlikely that lack of historical residence and pollution result in a crosssectional bias but it limits disentanglement of the effects on the level of $\mathrm{FEV}_{1}$ or the decline. Furthermore, not one of the three studies included data on time activity patterns. Commuting may account for a considerable proportion of the total exposure despite its short duration. ${ }^{8}$ The fact of assuming as negligible exposures occurring in sites other than residence may have underestimated the true associations. In any case, a further step ahead in new studies is required in order to incorporate estimations beyond residence, such as those occurring during commuting.

A formal assessment of communitylevel confounding is impossible, as these studies did not measure community-level factors. However, the larger number of geographical units in the English study and the multilevel modelling reduce the potential for confounding. Nevertheless, Forbes et al signal occupational exposures as a potential residual confounding in their study given the possible correlation between higher exposure to pollutants at work and higher levels of outdoor pollution. This seems unlikely not only because of the large size of the study, but also because while it is true that air pollution is correlated with poorer social conditions in the USA, in Europe the pattern is less clear. $^{910}$ Moreover, recent studies on current occupational exposures find a very weak effect on lung function. ${ }^{11}$

The authors speculate that the residual confounding by occupational exposures might explain the larger effect in men than women (about a $4 \%$ vs a $2 \%$ decrease per each $10 \mu \mathrm{g} / \mathrm{m}^{3}$ of $\left.\mathrm{PM}_{10}\right)$. In contrast, the authors of the SALIA study suggested that their stronger effect in comparison with previous studies was due to the fact that all their participants were women. There is a large body of literature about sex differences in the effects of smoking and also air pollution on lung function, without any agreement about their actual occurrence and if so its cause. Overall, susceptibility factors modifying adverse effects of air pollution on lung function are barely understood and need further investigation, which also applies to the unexpected funding of an increased risk for ex-smokers.

Forbes et al pointed out as one of the strengths of the study the inclusion of multiple pollutants since the role of specific pollutants or pollution sources is one of the unsolved questions that needs to be clarified. However, $\mathrm{PM}_{10}$ and $\mathrm{NO}_{2}$ were highly correlated (correlation coefficient $\sim 0.93$ ), given their common origin-that is, mainly road traffic-which precluded disentangling their effects. They also found an effect of $\mathrm{SO}_{2}$, whose correlation with the other pollutants is smaller due to a potential different origin. Unfortunately, the authors did not present any multipollutant model to see if there was an added effect of $\mathrm{SO}_{2}$. The effect obtained when pollutants were assessed one by one is probably an underestimation of the effect of the 
outdoor pollution mixture. Ozone in England does not seem to play a role in lung function.

Previous studies do not provide enough evidence to relate the effects of air pollution conclusively to one or the other specific spirometric measures, although there are some indications of larger effects on the markers of the small airways. ${ }^{4}$ Forbes et al provide data only on $\mathrm{FEV}_{1}$ and the $\mathrm{FEV}_{1} / \mathrm{FVC}$ ratio, showing no association for the ratio after adjusting for smoking and social class. Larger misclassification in the measurement of FVC than $\mathrm{FEV}_{1}$ in the general population studies may explain these results in part.

The larger size and improved exposure assessment in the study of Forbes et al reinforce the knowledge of a deleterious effect of current levels of air pollution on lung health in Europe. However, the cross-sectional nature of these studies does not solve important questions regarding the most relevant age period and exposure time windows of susceptibility. It is impossible to know if the effects on the lung function level in adulthood reflect growth deficits experienced during childhood and whether these subjects entered the lung function decline phase with a reduced lung function. The largest effects observed by Forbes et al were among the oldest people (>75 years). Nevertheless, this does not imply an effect during the decline phase since it could result from a cohort effect because of higher historical air pollution levels for this age group. It is possible that air pollution behaves like smoking in adulthood, which accelerates lung function decline, and that changes in smoking resulted in changes in the slope of decline. In any case, there is a need to extend follow-up studies to children and to perform large follow-up studies through to adolescence in order to quantify the magnitude of the effect of air pollution in accelerating lung function decline not only for a better knowledge of the origins of COPD, but also to assess the population impact of air pollution and the potential consequences of its reduction.

Competing interests: None declared.

Thorax 2009;64:645-646. doi:10.1136/thx.2009.115071

\section{REFERENCES}

1. Pope CA III, Dockery DW. Health effects of fine particulate air pollution: lines that connect. J Air Waste Manag Assoc 2006;56:709-42.
2. Romieu I, Castro-Giner F, Künzli N, et al. Air pollution oxidative stress and dietary supplementation: a review. Eur Resp J 2008;31:179-97.

3. Götschi T, Heinrich J, Sunyer J, et al. Long-term effects of ambient air pollution on lung function: a review. Epidemiology 2008;19:690-701.

4. Downs SH, Schindler C, Liu LJ, et al. Reduced exposure to PM10 and attenuated age-related decline in lung function. N Engl J Med 2007;357:2338-47.

5. Ackermann-Liebrich U, Leuenberger P, Schwartz J, et al. Lung function and long term exposure to air pollutants in Switzerland. Study on air pollution and lung diseases in adults (SAPALDIA) team. Am J Respir Crit Care Med 1997;155:122-9.

6. Schikowski T, Sugiri D, Ranft U, et al. Long-term air pollution exposure and living close to busy roads are associated with COPD in women. Respir Res 2005; 6:152

7. Forbes LJL, Kapetanakis V, Rudnicka AR, et al. Chronic exposure to outdoor air pollution and lung function in adults. Thorax 2009:64:657-63.

8. Hertel 0, Hvidberg M, Ketzel M, et al. A proper choice of route significantly reduces air pollution exposure-a study on bicycle and bus trips in urban streets. Sci Total Environ 2008;389:58-70.

9. Forastiere F, Stafoggia M, Tasco C, et al. Socioeconomic status, particulate air pollution, and daily mortality: differential exposure or differential susceptibility. Am J Ind Med 2007;50:208-16.

10. Généreux M, Auger N, Goneau M, et al. Neighbourhood socioeconomic status, maternal education and adverse birth outcomes among mothers living near highways. J Epidemiol Community Health 2008;62:695-700.

11. Sunyer J, Zock JP, Kromhout $\mathrm{H}$, et al. Lung function decline, chronic bronchitis and occupational exposures in young adults. Am J Respir Crit Care Med 2005;172:1139-45.

\section{Can traffic-related air pollution cause asthma?}

\section{John R Balmes}

Traffic-generated pollution contains particles and gases (eg, oxides of nitrogen) that are known to have health effects. ${ }^{1}$ Concentrations of pollutants emitted by motor vehicles are highest within $150 \mathrm{~m}$ of roadways and remain raised up to $300 \mathrm{~m}$ from roadways, but fall off markedly beyond that range. ${ }^{2}$ Exposure to the mixture of traffic-generated pollutants may be more relevant to human health than exposure to any single ambient air pollutant, making epidemiological investigations of traffic effects a key component of research into the public health impact of air pollution. However, assessment of exposure to traffic-related air pollution can be problematic. Exposure to traffic can be estimated with complex

Correspondence to: Dr J R Balmes, Department of Medicine, University of California, San Francisco and School of Public Health, University of California, Berkeley, California, USA; john.balmes@ucsf.edu dispersion models of pollutants from local freeway and non-freeway sources, but the data inputs required for such modelling are not always available. A frequently used simpler approach has been to estimate residential distance to roadways.

A number of studies have found an increased prevalence of asthma or asthma symptoms in children who live near roadways with high traffic counts. ${ }^{4-8}$ One large British study that focused on traffic within $150 \mathrm{~m}$ of children's homes found a gradient in risk that increased markedly with decreasing residential distance to a main road. ${ }^{7}$ A large study in southern California showed an increased prevalence of asthma and wheeze in children living within $75 \mathrm{~m}$ of a major roadway. ${ }^{9}$ Another study by Jerrett et al that analysed data from the same southern California cohort was able to demonstrate an association between the incidence of asthma and exposure to traffic-related pollution. ${ }^{10}$ A recent review summarised the evidence for traffic pollution as a risk factor for both asthma exacerbation and onset as strong. ${ }^{11}$

In contrast to the relatively rich literature for children, little has been published on the effects of traffic-related pollution on asthma in adults. Although several previous studies in adults with asthma have found that exposure to traffic-as measured by distance of residence from nearest major roadway-was associated with asthma symptoms, health care utilisation or decreased lung function, ${ }^{12-14}$ the study by Künzli and colleagues ${ }^{15}$ reported in this issue of Thorax is the first to show convincing evidence that exposure to traffic-related particulate matter increases the risk of adult-onset asthma (see page 664). When the paper by Künzli et al is taken together with the study by Jerrett et al, ${ }^{10}$ we now have evidence in both children and adults that traffic-related pollution can cause as well as exacerbate asthma.

Given the robust effects observed on asthma outcomes in other studies of both children and adults, it is somewhat surprising that distance to roadway was not associated with the risk of new-onset asthma in over 2700 non-smoking Swiss 